\title{
Large-scale chaos for arbitrarily small perturbations in nontwist Hamiltonian systems
}

\author{
G. Voyatzis, E.Meletlidou and S.Ictiaroglou \\ Department of Physics \\ University of Thessaloniki, Thessaloniki, 54006 GREECE \\ fax: +3031998037, email: voyatzis@auth.gr
}

Chaos,Soliton and Fractals, 14, 1179-1191, 2002.

\begin{abstract}
The overlapping of isochronous resonances of non-twist Hamiltonian systems can be studied by considering integrable models which result in a smooth connection of the homoclinic and heteroclinic manifolds called "reconnection". A complex net of separatrices is formed that depends on the number of the overlapped resonances and their characteristic type. One degree of freedom Hamiltonians are constructed that can describe efficiently the topological structure of nontwist systems. Applying the Melnivov's method, it is shown that, for arbitrarily small perturbations, the manifolds intersect transversely and chaotic behaviour spreads on the whole domain of the reconnected separatrices.
\end{abstract}

\section{Introduction}

For near integrable Hamiltonian systems of two degrees of freedom, of the form

$$
H=H_{0}\left(I_{1}, I_{2}\right)+\varepsilon H_{1}\left(I_{1}, I_{2}, w_{1}, w_{2}\right),
$$

where $I_{1}, I_{2} \in[\alpha, \beta] \subset \mathbb{R}, w_{1}, w_{2} \in \mathbb{S}^{1}$ and $\varepsilon$ is a small parameter, most of the fundamental theorems are applicable under the necessity of a non-degeneracy condition [1]. The required non-degeneracy is equivalent to the fact that the rotation number of $H_{0}$ is a monotonically increasing or decreasing function 
along the invariant circles of the unperturbed map, defined in a constant energy level $H_{0}\left(I_{1}, I_{2}\right)=h$, i.e

$$
\frac{d \rho\left(I_{1} ; h\right)}{d I_{1}} \neq 0 \quad, \quad \rho=\frac{\omega_{1}}{\omega_{2}}
$$

where $\omega_{i}=\partial H_{0} / \partial I_{i}$ are the frequencies of the system.

When the twist condition (1) is satisfied then the phase space of the integrable system is foliated by invariant tori filled with, generically, quasiperiodic trajectories. Under small perturbations, most of these tori persist while, near the resonant tori where the trajectories are periodic, stable and unstable periodic orbits bifurcate according to Poincaré-Birkhoff theorem [1]. The unstable ones are associated with stable and unstable manifolds that, generically, intersect transversely resulting to the formation of a chaotic set [2]. This behaviour is better understood and studied by considering the corresponding Poincaré map which is a perturbed twist map.

Both, the KAM theorem and the Poincaré-Birkhoff theorem are of ambiguous validity when (1) is not satisfied. The survival of quasiperiodic invariant curves seems again to be the dominant characteristic $[3,4,5]$, however the bifurcation scenario is different and varies from system to system. Howard and Hohs [6] showed the formation of vortices in a standard-like map while van der Weele and Valkering [7] showed the formation of dimerized chains in a quadratic area preserving map. Both cases appear when the rotation number presents an extremum and gives rise to the birth of two isochronous Poincaré-Birkhoff chains. Both phenomena are related to the overlapping of these chains, which takes place through a reconnection process.

Recent works reveal a variety of bifurcations and topological structures for non-twist maps. van der Weele and Valkering [8] constructed a mapping model to study the reconnection of four chains while Voyatzis and Ichtiaroglou [9] studied the case of three chains where the rotation number has also an inflection point. The radial non-twist maps, studied by Howard and Humphreys [10] and Petrisor [5], present also various cases of bifurcations for periodic orbits of period two or larger, while Simó [3] demonstrates some maps that give rise to the formation of "labyrinthic" invariant curves.

Nontwist bifurcations and reconnection can be studied in a framework of integrable Hamiltonians, which have the form of the Chirikov's pentulum system $[11,12]$ or by constructing integrable nontwist discrete maps. Perturbed nontwist systems and the transition to chaos are also of great importance since the nontwist condition is met in many problems of theoretical and physical interest e.g. in the Henon map [7, 13], tokamacks [14], underdamped oscillators [15] e.t.c. The transition to chaos has been studied through renormalization techniques by del Castillo-Negrete et al [16] while 
in Prado and Corso [17] chaos in the domain of reconnected manifolds is studied numerically by calculating a diffusion coefficient.

In this paper the generation of chaos under arbitrarily small perturbations is studied through the Poincaré-Melnikov-Arnold method or shortly addressed as the Melnikov method $[2,18]$. In section 2 we summarize and discuss some results obtained in [9] that refer to an integrable nontwist Hamiltonian model. In section 3, we show that nontwist phenomena can be studied by considering one degree of freedom systems with periodic-time dependent perturbations. In section 4 we calculate the Melnikov integral for some particular and representative systems and finally in section 5 , the generation of chaos is discussed.

\section{Integrable approximation of isochronous res- onances}

Following the integrable approximation, used by Carvalho and Almeida [12] to study the overlap of isochronous resonances, we consider Hamiltonian systems of the form

$$
H_{0}=H_{00}+k H_{01}=G_{1}\left(I_{1}\right)+G_{2}\left(I_{2}\right)+k f\left(I_{1}\right) \cos \left(p w_{2}-r w_{1}\right),
$$

where $I_{1}, I_{2} \in[\alpha, \beta] \subset \mathbb{R}, w_{1}, w_{2} \in \mathbb{S}^{1}$ and $p, r$ are integers. $H_{00}$ is nondegenerate when $\operatorname{det}\left|\partial^{2} H_{00} / \partial I_{1} \partial I_{2}\right| \neq 0$, and, it is isoenergetically nondegenerate when

$$
\operatorname{det}\left|\begin{array}{cc}
\frac{\partial^{2} H_{00}}{\partial I_{i} \partial I_{j}} & \omega_{i} \\
\omega_{j} & 0
\end{array}\right|=\omega_{2}^{2} \frac{\partial \omega_{1}}{\partial I_{1}}+\omega_{1}^{2} \frac{\partial \omega_{2}}{\partial I_{2}} \neq 0,
$$

where $\omega_{i}=\partial G_{i} / \partial I_{i}, i=1,2$ are the frequencies of $H_{00}$. Note that condition (3) is equivalent to (1) on the hypersurface of constant energy $h$. For $k=0$ the Poincaré map consists of invariant curves with rotation number $\rho\left(I_{1}, I_{2}\right)=\omega_{1} / \omega_{2}$. Then (3) guarantees that, under the effect of the term $k H_{01}$, the Poincare map in the neighbourhood of the resonance $p: r$ is a twist map and the resonant invariant curve breaks, generating a PoincaréBirkhoff chain with $r$ stable and $r$ unstable fixed points. We note that $H_{0}$ is integrable for $k \neq 0$ since it possesses a second integral of motion

$$
\Phi=p I_{1}+r I_{2} .
$$

By setting $G_{2}\left(I_{2}\right)=I_{2}$ and $G_{1}=\rho_{0} I_{1}+F\left(I_{1} ; \bar{a}\right)$, where $\rho_{0}=p / r$ is the particular resonance studied, (2) takes the form

$$
H_{0}=H_{00}+k H_{01}=I_{2}+\rho_{0} I_{1}+F\left(I_{1} ; \bar{a}\right)+k f\left(I_{1}\right) \cos \left(p w_{2}-r w_{1}\right),
$$


For this specific choice, $H_{00}$ is always degenerate, since $\partial^{2} H_{00} / \partial I_{2}^{2} \equiv 0$, but, generally, is isoenergetically nondegenerate. The functions $f, F$ are assumed to be analytic functions of $I_{1}$ and $\bar{a}$ denotes a parameter vector. In [9] $F\left(I_{1}\right)$ is assumed to be a polynomial function which corresponds to the Birkhoff normal form. This is not necessary but, on the other hand, it does not affect the genericity of the study. For system (5), the nondegeneracy condition (3) is the twist condition written as

$$
\frac{\partial \rho}{\partial I_{1}}=\frac{\partial^{2} F\left(I_{1} ; \bar{a}\right)}{\partial I_{1}^{2}} \neq 0
$$

where $\rho=\rho\left(I_{1}\right)$ is the rotation number of $H_{00}$. Therefore, when $F^{\prime}=$ $\partial F\left(I_{1} ; \bar{a}\right) / \partial I_{1}$ is a monotonically increasing (or decreasing) function and possesses a simple root for $I_{1}=I_{1}^{*}$ then the twist condition is satisfied and, for any constant energy hypersurface, $H_{00}$ possesses a $p: r$ resonant torus at $I_{1}=F^{\prime-1}(\bar{a}) . H_{0}$ has the form of a pendulum Hamiltonian (in the sense of Chirikov [11]), and for $k \neq 0$, a Poincaré-Birkhoff chain is formed on the plane $w_{2}=0(\bmod 2 \pi)$ with $r$ stable and $r$ unstable fixed points.

Isochronous resonances exist when $F^{\prime}\left(I_{1} ; \bar{a}\right)$ possesses more than one roots, say $I_{1}^{(i)}, i=1, . ., m$ and consecutive values of $i$ denote neighbor chains. Because of the continuity of $F^{\prime}, \rho\left(I_{1}\right)$ shows extrema between the roots and consequently, the twist condition (6) is violated. We should note that (6) is violated too when $\rho\left(I_{1}\right)$ has inflection points. However, despite the above degeneracy, the Poincaré-Birkhoff theorem is valid in a domain around the isochronous resonant tori when the resonant invariant curves are far from each other and condition (6) holds locally when

$$
\Delta I_{1}^{(i)}(\bar{a}, k)=\left|I_{1}^{(i)}-I_{i}^{(i+1)}\right|>C(k)
$$

where $C(k)$ is sufficiently large. Therefore, the parametric space $\mathcal{P}=\{\bar{a}, k\}$ is devided in two subsets $\mathcal{P}_{1}$ and $\mathcal{P}_{2}$ according to whether (7) is satisfied or not respectively. For $\bar{a} \in \mathcal{P}_{2}$ various degenerate bifurcation schemes can take place depending on particular properties of the functions $F$ and $f$. Such bifurcations are generic for any Hamiltonian system with nontwist domains [13]. For a critical value of $\Delta I_{1}^{(i)}$ (for some parameter values $\bar{a}=\bar{a}_{\text {rec }}$ and $k=k_{\text {rec }}$ ) the homoclinic manifolds of the isochronous chains are connected smoothly (separatrix reconnection). For the system (5) the reconnection condition is $[9]$

$$
S^{(i)}(\bar{a})=\int_{I_{1}^{(i)}}^{I_{1}^{(i+1)}}\left(\rho\left(I_{1} ; \bar{a}\right)-\rho_{0}\right) d I_{1}=\left.k \Delta H_{1}\right|_{P^{\prime}} ^{P} \quad 0<i<m
$$


where $P$ and $P^{\prime}$ denote unstable fixed points belonging in neighboring chains. We remark that the quantity $S^{(i)}$ is the area of the contour on the plane $\left(I_{1}, \rho\right)$ formed by the line $\rho=\rho_{0}$ and the curve $\rho\left(I_{1}\right)$ for $I_{1} \in\left[I_{1}^{(i)}, I_{1}^{(i+1)}\right]$ (e.g. the area, denoted by $R$ in figure 2).

Generally, nontwist bifurcations or separatrix reconnection do not take place for the same value of $\bar{a}$ for all isochronous resonances. A global reconnection of the $m$ chains is possible if the system of equations (8), $0<i<m$, has a solution for $\bar{a}$ and $k$. In this case a web of homoclinic and heteroclinic manifolds is formed connecting all the unstable fixed points. The reconnection web may be extended in a wide area of a constant energy hypersurface. We should remark that (5) is integrable for any $\bar{a}$ and $k$, and transversal manifold intersections are forbidden.

\section{One degree of freedom Hamiltonian repre- sentation}

In this section we show that the reconnection of isochronous resonances can be studied considering one-degree of freedom systems. This way is more convenient for calculating asymptotic solutions and applying Melnikov's method.

\subsection{Integrable systems}

We define new variables $J, I \in[\alpha, \beta] \subset \mathbb{R}, \theta, \phi \in \mathbb{S}^{1}$ and we consider the canonical transformation with generating function

$$
S=-\left(\frac{p}{r} w_{2}-w_{1}\right) J+w_{2} I
$$

Then, on any constant energy surface $H_{0}(J, I, \theta)=h$, the system (5) is described by the one degree of freedom Hamiltonian

$$
\bar{H}(J, \theta)=F(J ; \bar{a})+k f(J) \cos (r \theta)=h-I(J, \theta ; h) .
$$

The corresponding equations of motion are

$$
\begin{aligned}
\dot{J} & =r k f(J) \sin (r \theta), \\
\dot{\theta} & =F^{\prime}(J ; \bar{a})+k f^{\prime}(J) \cos (r \theta),
\end{aligned}
$$

where the prime denotes differentiation with respect to the action $J$. For $\theta=j \pi / r, j=0, . ., 2 r-1,2 r$ fixed points are obtained located at values of $J$ satisfying the equation

$$
F^{\prime}(J ; \bar{a})+(-1)^{j} k f^{\prime}(J)=0 .
$$


Let $J_{1}, J_{2}, \ldots, J_{m}$ be the roots of eq. (11) and $P(i, j), 0<i \leq m, 0 \leq j<2 r$ denotes the fixed point $(J, \theta)=\left(J_{i}, j \pi / r\right)$. The stability of the $2 \mathrm{rm}$ fixed points is studied in [9] and the results obtained there can be applied directly to system (9). When condition (7) is satisfied then $m$ isochronous PoincaréBirkhoff chains appear and they are assigned by their particular sets of fixed points $C^{(i)}=\{P(i, j), 0 \leq j<2 r\}, i=1, . ., m$. For two consecutive chains, $C^{(i)}$ and $C^{(i+1)}$, we distinguish two different cases, called "Type I" and "Type II", according to the stability distribution of their fixed points. For chains of "Type I" the fixed points, $P(i, j)$ and $P(i+1, j)$ are of different stability for all $j \leq r$, while, for chains of "Type II", $P(i, j)$ and $P(i+1, j)$ are of the same stability type for all $j \leq r$. In [9] it is shown that when the restriction $\left|\partial^{2} \bar{H} / \partial J^{2}\right|_{J=J_{i}}>k\left|\partial^{2} f / \partial J^{2}\right|_{J=J_{i}}, \forall i \leq m$, is satisfied, the stability distribution depends on the function $f(J)$ and we have

$$
\begin{array}{ll}
\text { "Type I" for } & f\left(J_{i}\right) f\left(J_{i+1}\right)>0, \\
\text { "Type II" for } & f\left(J_{i}\right) f\left(J_{i+1}\right)<0 .
\end{array}
$$

Figure 1 illustrates the two different stability distribution types. Reconnection of the chains $C^{(i)}$ and $C^{(i+1)}$ happens for $\bar{a}=\bar{a}_{\text {rec }}$ such that the chains are on the same energy level. Assuming that the fixed point $P(i, 0) \in C^{(i)}$ is unstable, the reconnection condition is written

$$
F\left(J_{i+1} ; \bar{a}_{r e c}\right)-F\left(J_{i} ; \bar{a}_{r e c}\right)=k\left(f\left(J_{i}\right) \pm f\left(J_{i+1}\right)\right),
$$

where the plus sign refers to "type I" and the minus to "type II". The condition (13) is consistent with the general condition (8). The simplest case is when the rotation number possesses a simple extremum and, thus, (11) provides two isochronous chains. Figure 2 presents the topological structures obtained for the two types. We should note that the chains $C^{(1)}$ and $C^{(2)}$ (first row of figure 2) survive up to the reconnection. This is also true for the case of three isochronous chains of type I, but for the type II additional bifurcations are obtained between the reconnection and the splitting of the isochronous chains [9]. For radial nontwist discrete maps, studied by Howard and Humpheys [10], the structure of "type I" is apparent, while "type II" is formed for period-two. The maps obeying a reversing symmetry, studied by Petrisor [5], show the structure of the first or second type when the resonance order is an odd or an even number respectively. For systems (9) both types can be constructed by satisfying the condition (12) which is independent of the resonance order and the number of isochronous chains.

For more than two chains, a global reconnection is possible when (13) is satisfied for all neighbor chains. Let us consider the system

$$
H=J^{5} / 5-2 J^{3} / 3+a J+k f(J) \cos \theta .
$$


The function $F^{\prime}(J ; a)$ shows three extrema, so the formation of four isochronous chains is possible. By setting $f(J)=1$, according to (12) only the case of "type I" is possible and the fixed points are located at $J= \pm \sqrt{1 \pm \sqrt{1-a}}$. By taking into account the reconnection condition for all unstable points we find that global reconnection happens for $a_{r e c}=4 / 9$ and $k_{r e c} \simeq 0.145$. If we set $f(J)=J$ the unstable fixed points are located at $J_{1,4}= \pm \sqrt{1+\sqrt{1-a-k}}$ and $J_{2,3}= \pm \sqrt{1-\sqrt{1-a+k}}$. Since $J_{1}, J_{2}$ are both positive, the chains $C^{(1)}$ and $C^{(2)}$ are of "type I" and, also, the same holds for the chains $C^{(3)}$ and $C^{(4)}$ because $J_{3}$ and $J_{4}$ are both negative. However, $C^{(2)}$ and $C^{(3)}$ are of "type II". A global reconnection is possible for this "mixed" type too, and, using (13), we find that $a_{r e c}=k_{r e c}=5 / 18$. Figure 3 shows the phase portraits of the above reconnection. We remark that a global reconnection is possible when the system of equations (13), for $i=1,2, \ldots, m$, has solutions in the parameter space $(\bar{a}, k)$ and therefore, the dimension of $\bar{a}$ should be at least equal to $m-2$.

\subsection{Nonintegrable perturbations}

We perturb the integrable Hamiltonian (2) by adding the perturbation term $\varepsilon \tilde{H}_{1}\left(I_{1}, I_{2}, w_{1}, w_{2}\right)$, where $\varepsilon$ is a small parameter and $\tilde{H}_{1}$ is $2 \pi$-periodic with respect to the angles. For a non-degenerate system and for $\varepsilon \neq 0$ the homoclinic manifolds of the unstable periodic orbits, which either exist and merge smoothly for $\varepsilon=0$ or are generated by the perturbation itself, intersect transversely. In general, such intersections prove to exist when a certain Melnikov function has simple zeros. This property can be also used to study the effect of a perturbation on the reconnected isochronous manifolds. Veerman and Holmes [19] generalized Melnikov's method for 2 degrees of freedom systems of coupled pendula by reducing the original two degrees of freedom perturbed system. Following their method, we consider the perturbed system

$$
H=I+F(J ; \bar{a})+k f(J) \cos (r \theta)+\varepsilon \tilde{H}_{1}(J, I, \theta, \phi),
$$

We restrict to a three dimensional energy surface $H(J, I, \theta, \phi)=h$ and, applying the implicit function theorem for small $\varepsilon$, we solve (15) for the action $I$

$$
I=h-K(J, \theta)-\varepsilon \tilde{H}_{1}(J, \theta, \phi ; h-K(J, \theta))+O\left(\varepsilon^{2}\right),
$$

where $K(J, \theta)=F(J ; \bar{a})+k f(J) \cos (r \theta)$. By taking into account that $\phi=$ $t+O(\varepsilon)$, the equations for $J$ and $\theta$ are written as

$$
\dot{J}=-\frac{\partial K}{\partial \theta}-\varepsilon \frac{\partial \tilde{H}_{1}(J, \theta, t ; h)}{\partial \theta}+O\left(\varepsilon^{2}\right),
$$




$$
\dot{\theta}=\frac{\partial K}{\partial J}+\varepsilon \frac{\partial \tilde{H}_{1}(J, \theta, t ; h)}{\partial J}+O\left(\varepsilon^{2}\right) .
$$

Changing the time-scale $(t \rightarrow r t)$, it results that, up to order $\varepsilon$, the effect of the perturbation in (15) can be studied by considering the periodically time-dependent one degree of freedom Hamiltonian

$$
\bar{H}=\bar{H}_{0}+\varepsilon \tilde{H}_{1}=F(J ; \bar{a})+k f(J) \cos (\theta)+\varepsilon \tilde{H}_{1}(J, \theta, t ; h) .
$$

The above reduction means that, instead of using system (15), we can study the perturbed orbits by considering (9) and adding periodically time dependent perturbations of the form $\varepsilon H_{1}(J, \theta, t)$.

\section{Perturbed reconnected orbits and genera- tion of chaos}

In this section we consider systems of the form (18) in order to study the generation of transverse homoclinic and heteroclinic intersections by applying Melnikov's method. We consider the Melnikov integral, which, for periodically-time dependent one degree of freedom Hamiltonians, takes the form [18]

$$
M\left(t_{0}\right)=\int_{-\infty}^{\infty}\left[\bar{H}_{0}, \tilde{H}_{1}\right]_{0} d t
$$

where $[,]_{0}$ denotes the Poisson bracket and the integral is evaluated on the unperturbed homoclinic or heteroclinic trajectories. When $M\left(t_{0}\right)$ has simple zeros then the unstable manifolds intersect transversely for any small $\varepsilon \neq 0$, and according to the Birkhoff-Smale theorem, a chaotic set is formed.

We consider the interaction of two isochronous chains by considering particular representative systems. In [3] the same systems (but for fixing $k=1$ ) have been derived to describe the flow of some discrete non-twist maps.

\subsection{Reconnection of "type I"}

We construct an appropriate integrable system, exhibiting the expedient behaviour, by considering $F(J)$ to be a cubic polynomial and $f(J)=1$ that ensures stability distribution of "type I"

$$
\bar{H}_{0}=a J-J^{3} / 3+k \cos \theta,
$$

where $a, k$ are constant parameters. On the plane $(\theta, J)$ we consider the following representative fixed points

$$
\begin{array}{lll}
P_{1}^{U}=(-\pi, \sqrt{a}) & P_{2}^{S}=(0, \sqrt{a}) & P_{3}^{U}=(\pi, \sqrt{a}), \\
P_{4}^{S}=(-\pi,-\sqrt{a}) & P_{5}^{U}=(0,-\sqrt{a}) & P_{6}^{S}=(\pi,-\sqrt{a}) .
\end{array}
$$


The superscript $S$ or $U$ denotes whether the fixed point is stable or unstable respectively. We should note that all unstable fixed points are saddles. For $a<0$, the topology of the system is shown in the bottom row of figure 2 . The above fixed points appear after a saddle-center bifurcation for $a=0$. Then a pair of dimerized islands or meanders is formed up to $a=a_{r e c}$, where the islands become sufficiently large to touch each other. This is the stage of reconnection of the asymptotic manifolds and is illustrated in figure 4(a). The homoclinic orbit (A) that connects $P_{1}$ and $P_{3}$ and the two heteroclinic orbits (B) and (C) that connect $P_{5}$ with $P_{1}$ and $P_{3}$ respectively are shown. The reconnection occurs when (13) is satisfied, i.e. for

$$
k=\frac{2}{3} a^{3 / 2} .
$$

By substituting (21) in (20) we get a parametric solution for the orbits (A), (B) and (C) by solving the equation:

$$
J^{3}-3 a J-2 a^{3 / 2} \cos \theta=0 .
$$

After some calculations we find

$$
\begin{array}{ll}
J(\theta)=2 \sqrt{a} \cos (\theta / 3) & (A), \\
J(\theta)=-\sqrt{a}(\cos (\theta / 3)+\sin (\theta / 3)) & (B), \\
J(\theta)=-\sqrt{a}(\cos (\theta / 3)-\sin (\theta / 3)) & (C) .
\end{array}
$$

The corresponding solutions $\theta(t)$ can be found from the equation of motion for $\theta$ which reads

$$
\int \frac{d \theta}{1-(1 / a) J(\theta)^{2}}=a\left(t-t_{0}\right)
$$

By calculating the above integral for the three orbits and solving with respect to $\theta$, we get

$$
\theta(t)=W\left(\frac{a}{\sqrt{3}}\left(t-t_{0}\right)\right)
$$

where $t_{0}$ is the arbitrary initial time and

$$
\begin{array}{ll}
W(t)=-3 \arctan (\sqrt{3} \tanh (t)) & (A), \\
W(t)=3 \arctan \left(-\frac{\sqrt{3}}{e^{2 t}+1}\right) & (B), \\
W(t)=3 \arctan \left(\frac{\sqrt{3}}{e^{-2 t}+1}\right) & (C) .
\end{array}
$$

Knowing the homoclinic and heteroclinic solutions, we can calculate the Melnikov integral (19) to study the effect of a particular perturbation. We take as an example a simple perturbation term of the form

$$
\tilde{H}_{1}(J, \theta, t)=J \cos (\omega t)
$$


where $\omega$ is the frequency of the perturbation. The Poisson bracket in (19) is

$$
\left[\bar{H}_{0}, \tilde{H}_{1}\right]_{0}=-k \sin \theta(t) \cos (\omega t)=-k \sin W\left(t^{\prime}\right) \cos \left(\frac{\sqrt{3}}{a} \omega\left(t^{\prime}+t_{0}\right)\right),
$$

where $t^{\prime}=(a / \sqrt{3})\left(t-t_{0}\right)$. By expanding the cosine term, the Melnikov integral takes the form:

$$
M\left(t_{0}\right)=2 \sqrt{\frac{a}{3}}\left(M_{1} \sin \left(\omega t_{0}\right)-M_{2} \cos \left(\omega t_{0}\right)\right)
$$

where

$$
M_{1}=\int_{-\infty}^{\infty} Q(t) \sin \left(\omega^{\prime} t\right) d t, \quad M_{2}=\int_{-\infty}^{\infty} Q(t) \cos \left(\omega^{\prime} t\right) d t
$$

and $Q(t)=\sin (W(t)), \omega^{\prime}=(\sqrt{3} / a) \omega$. We observe that the calculation of $M\left(t_{0}\right)$ is associated to the particular homoclinic and heteroclinic solutions through the dimensionless function $Q(t)$, which is shown in figure 5. In the next paragraphs we use the notation

$$
\mu(x)=\int_{0}^{\infty}|Q(t)| \sin (x t) d t .
$$

For the cases studied below, the corresponding functions $\mu(x)$ are shown in figure 6 .

\subsubsection{Orbit (A)}

The following properties hold

$$
Q(-t)=-Q(t) \quad \text { and } \quad Q(t)<0, \forall t>0 .
$$

Thus, it comes out that $M_{2}=0$ and

$$
M_{1}=2\left(\int_{0}^{t_{m}} Q(t) \sin \left(\omega^{\prime} t\right) d t+\int_{t_{m}}^{\infty} Q(t) \sin \left(\omega^{\prime} t\right) d t\right)
$$

where $t_{m}=\tanh ^{-1}(1 / 3)=\ln (2) / 2$ is the position of the extremum of $Q(t)$ for $t>0$. Conclusively, the first integral is finite while the second one converges since $Q(t)$ is a positive and monotonically decreasing function towards zero for $t \geq t_{m}$, and, also, $\left|\sin \left(\omega^{\prime} t\right)\right|$ is bounded. Thus, we get

$$
M\left(t_{0}\right)=4 \sqrt{\frac{a}{3}} \mu\left(\omega^{\prime}\right) \sin \left(\omega t_{0}\right) .
$$




\subsubsection{Orbits (B) and (C)}

By considering a time shift $t^{\prime}=t-t_{m}, Q\left(t^{\prime}\right)$ becomes an odd function of $t^{\prime}$ for both orbits and

$$
-Q\left(t^{\prime}\right)_{(B)}=Q\left(t^{\prime}\right)_{(C)}>0 \forall t^{\prime}>0,
$$

where subscripts $(\mathrm{B})$ and $(\mathrm{C})$ denote the particular orbit. As in the previous case $M_{2}=0$. Also $\left|Q\left(t^{\prime}\right)\right|$ monotonically decreases towards zero $\forall t^{\prime}>0$ and thus $M_{1}$ converges to $\mu\left(\omega^{\prime}\right)$. The Melnikov function takes the form

$$
M\left(t_{0}\right)=\mp 4 \sqrt{\frac{a}{3}} \mu\left(\omega^{\prime}\right) \sin \left(\omega\left(t_{0}+t_{m}\right)\right),
$$

where signs $(-,+)$ refer to orbits $(\mathrm{B})$ and $(\mathrm{C})$ respectively.

\subsection{Reconnection of "type II"}

Similarly to the previous case we select $F(J)$ to be cubic polynomial and, in order to satisfy condition (12) for stability distribution of "type II", we set $f(J)=J$

$$
\bar{H}_{0}=a J-J^{3} / 3+k J \cos (\theta),
$$

where $a, k$ are parameters. For $a \pm k>0$ we have the following representative fixed points $(\theta, J)$ for the two isochronous chains

$$
\begin{array}{lll}
P_{1}^{U}=(-\pi, \sqrt{a-k}) & P_{2}^{S}=(0, \sqrt{a+k}) & P_{3}^{U}=(\pi, \sqrt{a-k}), \\
P_{4}^{U}=(-\pi,-\sqrt{a-k}) & P_{5}^{S}=(0,-\sqrt{a+k}) & P_{6}^{U}=(\pi,-\sqrt{a-k}) .
\end{array}
$$

In this case, according to (13), reconnection is obtained for $a=k$. Then the unstable fixed points $P_{1}$ and $P_{3}$ (which are saddles) coincide with $P_{4}$ and $P_{5}$ respectively forming the unstable points $P_{14}=(-\pi, 0)$ and $P_{35}=(\pi, 0)$ shown in figure $4 \mathrm{~b}$ with their asymptotic orbits (A), (B) and (C). Following the same steps as in the previous case, the solutions for the above orbits are found to be

$$
\begin{array}{rlrl}
(\mathrm{A}) & J=0 & \theta(t) & =2 \arctan \left(a\left(t-t_{0}\right)\right), \\
(\mathrm{B}, \mathrm{C}) & J= \pm \sqrt{3 a(1+\cos \theta)} & \theta(t)=-2 \arctan \left(2 a\left(t-t_{0}\right)\right) .
\end{array}
$$

Generally the term $\varepsilon \tilde{H}_{1}(J, \theta, t)$, added to (32), perturbs all the asymptotic orbits. However if $J$ is a factor of $\tilde{H}_{1}$ then for $\varepsilon \neq 0$ the orbit (A) is invariant. For example, under the perturbation

$$
\tilde{H}_{1}(J, \theta, t)=J \cos (\omega t) \quad(\text { type IIa }),
$$


two chaotic regions are expected which are separated by the invariant curve $J=0$, while the perturbation

$$
\tilde{H}_{1}(J, \theta, t)=\cos \theta \cos (\omega t) \quad(\text { type IIb) }
$$

should produce a chaotic set formed by the intersections of all the asymptotic orbits $(\mathrm{A}),(\mathrm{B})$ and $(\mathrm{C})$.

We mentioned above that the fixed point $P_{14}\left(\right.$ or $\left.P_{35}\right)$ is formed by the collision of two saddles. Thus $P_{14}$ is a "double saddle" which continuation is not guaranteed under the perturbation. Therefore, the Melnikov's method is not applicable in this case.

\section{Homoclinic and heteroclinic chaos}

The Melnikov integrals (30) and (31), obtained in the previous section for the reconnection of "type I", shows a sinusoidal variation in respect to $t_{0}$ with amplitude

$$
M_{0} \propto \sqrt{a} \mu(\omega ; a),
$$

which is related to the maximum distance $d_{0}$ of the stable and unstable manifolds in the perturbed systems [18] as

$$
d_{0}=\varepsilon \frac{M_{0}}{\left|\nabla H_{0}\right|}+O\left(\varepsilon^{2}\right)
$$

The parameter $a$ inserts in $\mu\left(\omega^{\prime}\right)$ as a frequency scaling factor. For all cases, $\mu\left(\omega^{\prime}\right)$ has one maximum of $O(1)$ and when $a$ is nonzero and finite, $\mu\left(\omega^{\prime}\right)$ is zero only for $\omega=0$ (integrable case) and for $\omega \rightarrow \infty$. Therefore, $M\left(t_{0}\right)$ possesses simple zeros for

$$
t_{0}=\frac{n \pi-\phi_{0}}{\omega}, \quad n= \pm 1, \pm 2, \ldots
$$

where $\phi_{0}=0$ for the homoclinic orbit (A) and $\phi_{0}=\omega t_{m}$ for the heteroclinic orbits (B) and (C).

Conclusively, homo-heteroclinic chaos is generated for arbitrarily small $\varepsilon \neq 0$ along the reconnected manifolds of "type I". Figure 7a shows the evolution of the homoclinic and heteroclinic manifolds, given by (23) and shown in figure 4a, under the perturbations considered in the previous section. After few intersections with the Poincaré section, defined by the period $T=2 \pi / \omega$, the expected splitting and the formation of tangles is apparent. We have used a quite large perturbation value $\varepsilon=0.1$ for illustration 
reasons, however, transverse intersections and tangles appear for arbitrarily small perturbations.

For the reconnection of "type II", although transversal intersections can not been shown by the Melnikov's method, we observe, similarly to the previous case, the formation of tangles as it is shown in figures $7 \mathrm{~b}$ and $7 \mathrm{c}$. The first case (type IIa) corresponds to the perturbation (34) and the tangles are separated by the invariant asymptotic (A). For the second case (type IIb), where the perturbation (35) is used, all the asymptotic orbits that correspond to the double-saddle fixed point are entangled forming a wide chaotic region.

Figure 8 shows the corresponding Poincaré sections for $\varepsilon=0.01$. Chaos is apparent almost everywhere in the domain of the separatrix splitting. For the non-generic case of type IIa, the invariant curve at $J=0$ is a boundary curve that prevents the mixing of the upper and lower chaotic domains. The perurbation also causes the breakup of the invariant curves of the secondary resonances 1:1, 2:1 e.t.c. We note that when the homo(hetero)clinic orbits are approached in the integrable system, the secondary rotation number, inside the island, tends to infinity. Thus many secondary resonances are located in the neigbourhood of the reconnection that contribute to the widening of the chaotic zone when the perturbation increases. In figure 8a the secondary resonance $2: 1$ is still outside of the reconnection domain but in figures $8 \mathrm{c}$ and $8 \mathrm{~d}$ the secondary resonance $2: 1$ has been overlapped by the main chaotic zone. It is remarkable to note the formation of the isolated island "I" shown in figure $8 \mathrm{~b}$ that does not seem to correspond to any secondary resonance and is located in the middle of the chaotic zone along the homoclinic branch.

The amplitude $\delta$ of the islands, which form the Poincaré-Birkhoff isochronous chains, depends on the parameter $k$. For the twist Chirikov's oscillator is $\delta \sim \sqrt{k}$. For the systems studied, we get

$$
\text { type I }: \delta=4 \sqrt{a}=4 k^{1 / 3} \quad \text { type II }: \delta=2 \sqrt{3 a}=2 \sqrt{3} k^{1 / 2} .
$$

Even for large values of $k$, the reconnection condition (13) is satisfied by some value of the parameter vector $\bar{a}$. Also when a large number of isochronous resonances is reconnected, then a web of reconnected malifolds is formed with arbitrarily large width $\delta$. In this case, small perturbations can produce large scale chaos along the "reconnection" web.

\section{Conclusions}

Starting from a class of degenerate Hamiltonians of two degrees of freedom we derived a form of equivalent one-degree of freedom systems with periodic time dependence. In the integrable framework the reconnection of 
isochronous Poinaré-Birkhoff chains can be studied in the absence of chaos. We distinguish two main types for the isochronous chains according to the stability distribution of their fixed points, which lead to different topological structures in the reconnection stage. A global reconnection of more than two chains of the same or different type is also possible. In our model the independent functions $F(J ; \bar{a})$ and $f(J)$ are associated directly with the number of isochronous chains (of an arbitrary resonance order) and their stability type respectively.

We mostly concentrate our study to the stage of reconnection. The nontwist bifurcations close to the reconnection condition, do not obey a particular scenario. Systems of type I and type II exhibit different bifurcations and this is also the case when the number of the reconnected chains is greater than two. For example, the reconnection of two chains of "type I" is associated with a saddle-center bifurcation and the formation of dimerized chains, while the reconnection of three chains of the same type is associated with "pitchfork" and "inverse pitchfork" bifurcations, since in this case the rotation number has an inflection point [9].

Performing a study of the isochronous chain reconnection in the integrable system we obtain a web of homoclinic and heteroclinic manifolds that are joined smoothly. We study the effect of perturbations by adding timedependent terms in the integrable one-degree of freedom Hamiltonian. Such perturbations describe, up to order $O(\varepsilon)$, a perturbed two degrees of freedom system and this approximation is sufficient for the study of possible transversal intersection of the invariant manifolds by using Melnikov's method. For the reconnection of "type I" the Melnikov integral has simple zeros and thus the existence of transversal intersections and, furthermore, the formation of a chaotic region around the reconnection domain, is proven. However, Melnikov's method is not applicable for the reconnection of "type II" where double-saddles appear. But also in this cases, the formation of tangles, observed numerically, indicate the existence of transversal intersections.

Acknowledgements: This work has been partially supported by scientific programme PENED-99, No 1958/100164, Greece (EM).

\section{References}

[1] V.I. Arnol'd, V.V. Kozlov and A.I. Neishtadt, Mathematical aspects of classical and celestial Mechanics, in Dynamical Systems III, ed. V.I. Arnol'd, Springer-Verlag: Berlin (1987). 
[2] S. Wiggins, Global bifurcations and chaos: Analytical methods, Springer, New York, (1990).

[3] C. Simó, Invariant curves of analytic perturbed nontwist area preserving maps, Regular and Chaotic Dynamics, 3, 180-195 (1998).

[4] A. Delshams and R. Llave, KAM theory and a partial justification of Greene's criterion for non-twist maps, Siam J. Math. anal.,31, 12351269 (2000).

[5] E. Petrisor, Nontwist Area preserving maps with reversing symmetry group, Int. J. of Bifurcation and Chaos, 11, 497-511 (1999).

[6] J.E. Howard and S.M. Hohs, Stochasticity and reconnection in Hamiltonian systems, Phys. Rev. A29, 418-421 (1984).

[7] J.P. van der Weele, T.P. Valkering, H.W. Capel, and T. Post, The birth of twin Poincaré-Birkhoff chains, Physica 153A, 283-294 (1988).

[8] J.P. van der Weele and T.P. Valkering, The birth process of periodic orbits in non-twist maps, Physica 169A, 42-72 (1990).

[9] G. Voyatzis and S. Ichtiaroglou, Degenerate bifurcations of resonant tori in hamiltonian systems, Int. J. of Bifurcation and Chaos, 9, 849-863 (1999).

[10] J.E. Howard and J. Humpherys, Nonmonotonic twist maps, Physica D80, 256-276 (1995).

[11] B.V. Chirikov, A universal instability of many-dimensional oscillator systems, Phys. Rep. 52, 362-418 (1979).

[12] R. Egydio de Carvalho and A.M. Ozorio de Almeida, Integrable approximation to the overlap of resonances, Phys. Lett. A162, 457-463 (1992).

[13] H.R. Dullin, J.D. Meiss and D. Sterling, Generic twistless bifurcations, Nonlinearity 13, 203-224 (2000).

[14] G.A. Oda and L.I. Caldas, Dimerized island chains in Tokamaks, Chaos, Solitons \& Fractals 5, 15-23 (1995).

[15] S.M. Soskin, D.G. Luchinsky,R. Mannella, A.B. Neiman and P.V.E. McClintock, Zero-dispersion nonlinear resonance, Int. J. Bifurcation and Chaos 7, 923-936 (1997). 
[16] D. del-Castillo-Negrete, J.M. Greene and P.J. Morrison, Renormalization and transition to chaos in area preserving nontwist maps, Physica 100D, 311-329 (1997).

[17] S.D. Prado and G. Corso, Manifold reconnection and diffusion in strong chaos, Physica 142D, 217-230 (2000).

[18] Guckenheimer, J. \& Holmes P., 1983, Nonlinear oscillations, dynamical systems and bifurcations of vector fields, Springer, N.Y. (1983)

[19] P. Veerman and P. Holmes, The existence of arbitrary many distinct periodic orbits in 2DoF hamiltonian systems, Physica D14, 177-192 (1985). 


\section{Caption of figures}

Figure 1. The two types of isochronous chains distinguished by the stability distribution of their fixed points.

Figure 2. The main topological structures when the rotation number possesses a single local extremum.

Figure 3. Reconnection of four isochronous resonances of "type I" (left) and of mixed type (right).

Figure 4. Reconnected manifolds for "type I" (left) with $\gamma=\sqrt{4 a}$ and "type II" (right) with $\gamma^{\prime}=\sqrt{3 a}$.

Figure 5. Graph of the function $Q(t)=\sin \theta^{\prime}(t)$ of the homoclinic and heteroclinic solutions for the case of "type I".

Figure 6. The function $\mu(x)$ that contributes to the Melnikov's integral.

Figure 7. The evolution of the initial homoclinic and heteroclinic orbits after 3 intersections with the Poincaré section. Blue, red and green colour presents orbits (A),(B) and (C) respectively. (a) Type I, (b) Type IIa and (c) Type IIb.

Figure 8. The Poincaré section for the examined systems and for $a=1$, $k=k_{\text {rec }}, \omega=1$ and $\varepsilon=0.01$. a) Type I, b) magnification of the area around the unstable fixed point at the center of (a), c) Type IIa, where only the upper chaotic region is shown, d) Type IIb. 


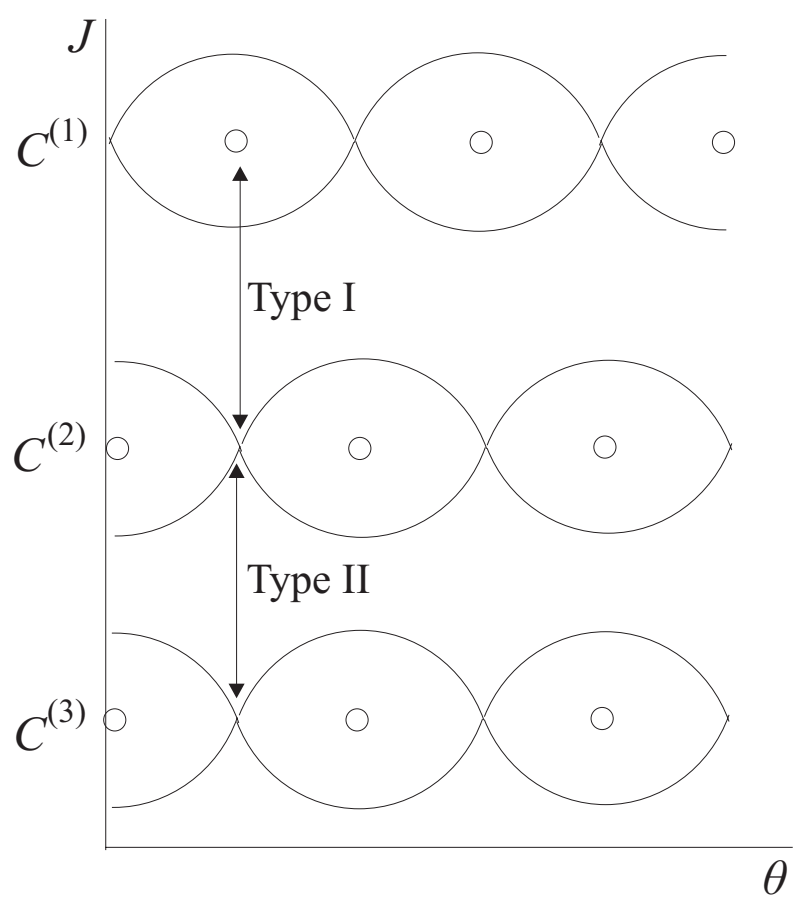

Figure 1: The two types of isochronous chains distinguished by the stability distribution of their fixed points. 


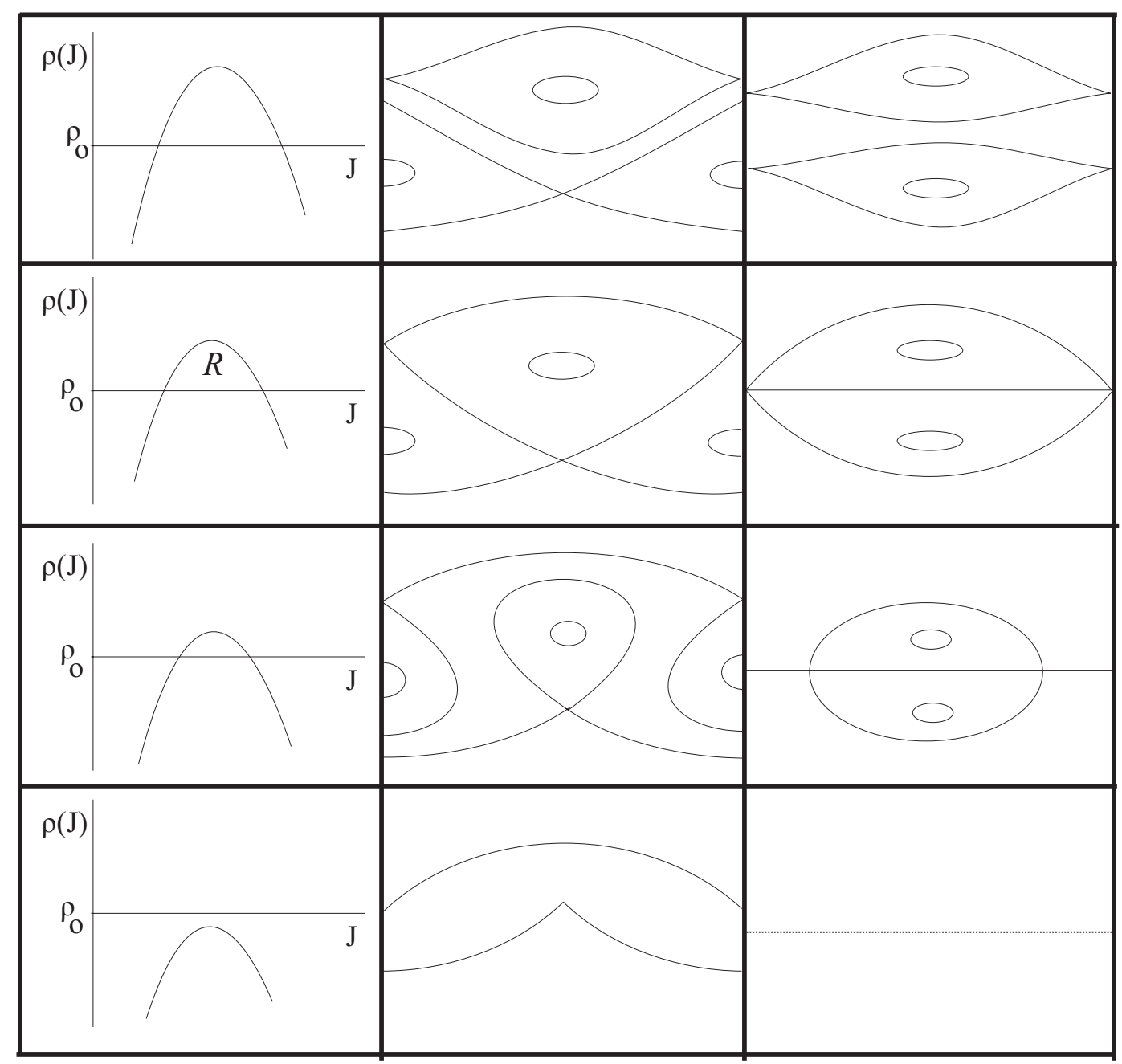

Figure 2: The main topological structures when the rotation number possesses a single local extremum. 

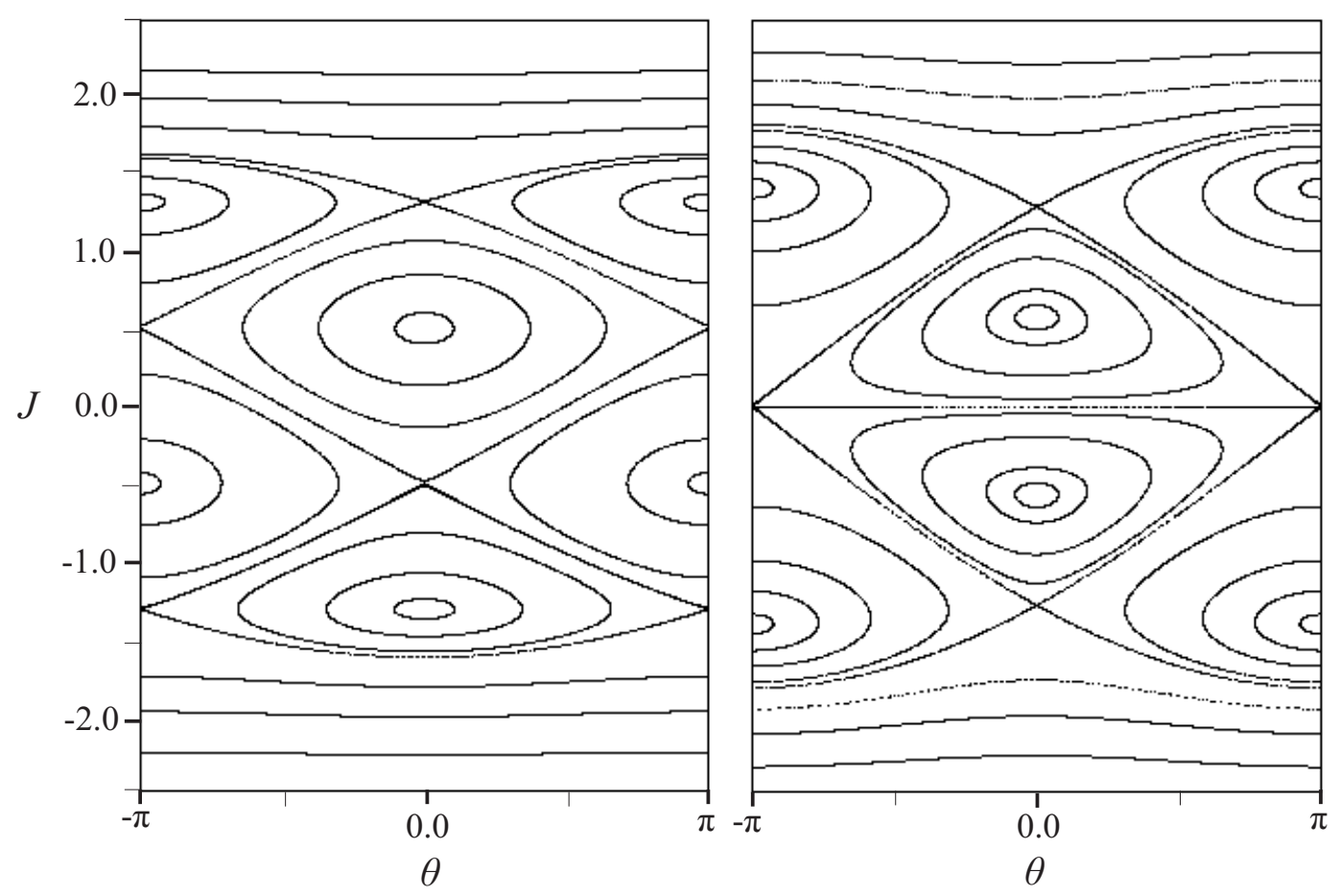

Figure 3: Reconnection of four isochronous resonances of "type I" (left) and of mixed type (right).

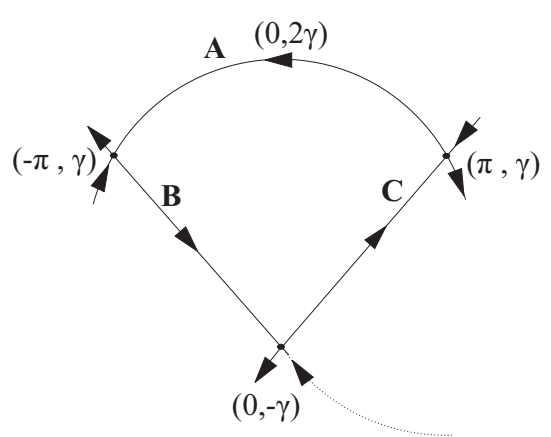

(a) Type I

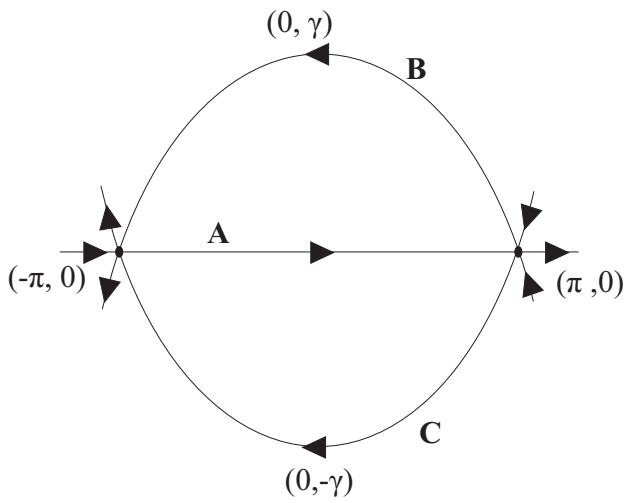

(b) Type II

Figure 4: Reconnected manifolds for "type I" (left) with $\gamma=\sqrt{4 a}$ and "type II" (right) with $\gamma^{\prime}=\sqrt{3 a}$. 


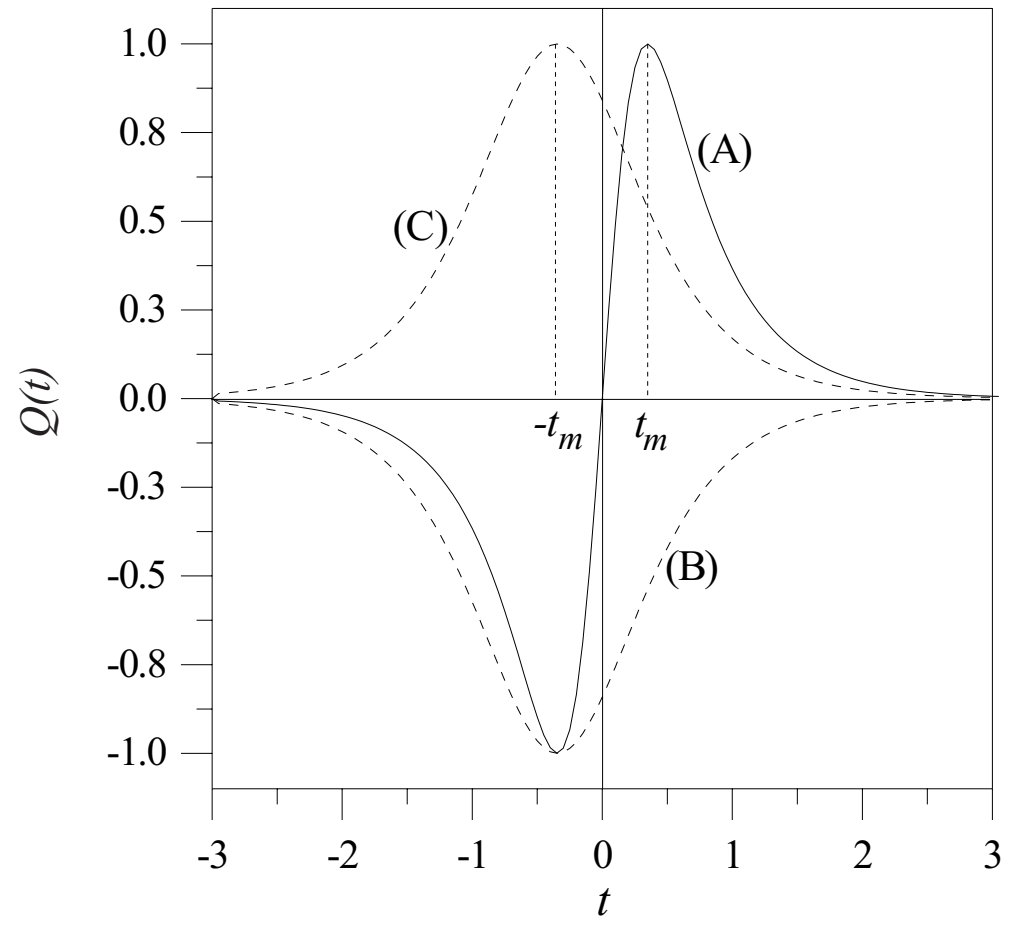

Figure 5: Graph of the function $Q(t)=\sin \theta^{\prime}(t)$ of the homoclinic and heteroclinic solutions for the case of "type I". 


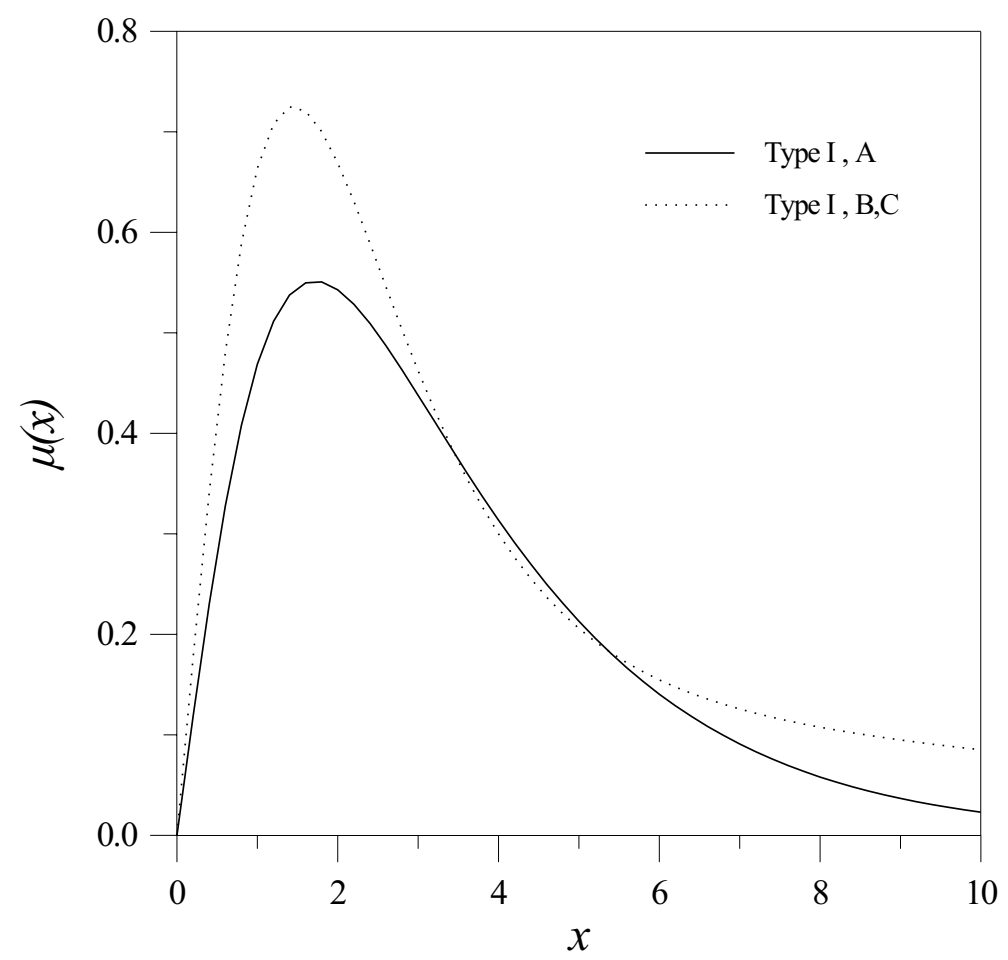

Figure 6: The function $\mu(x)$ that contributes to the Melnikov's integral. 

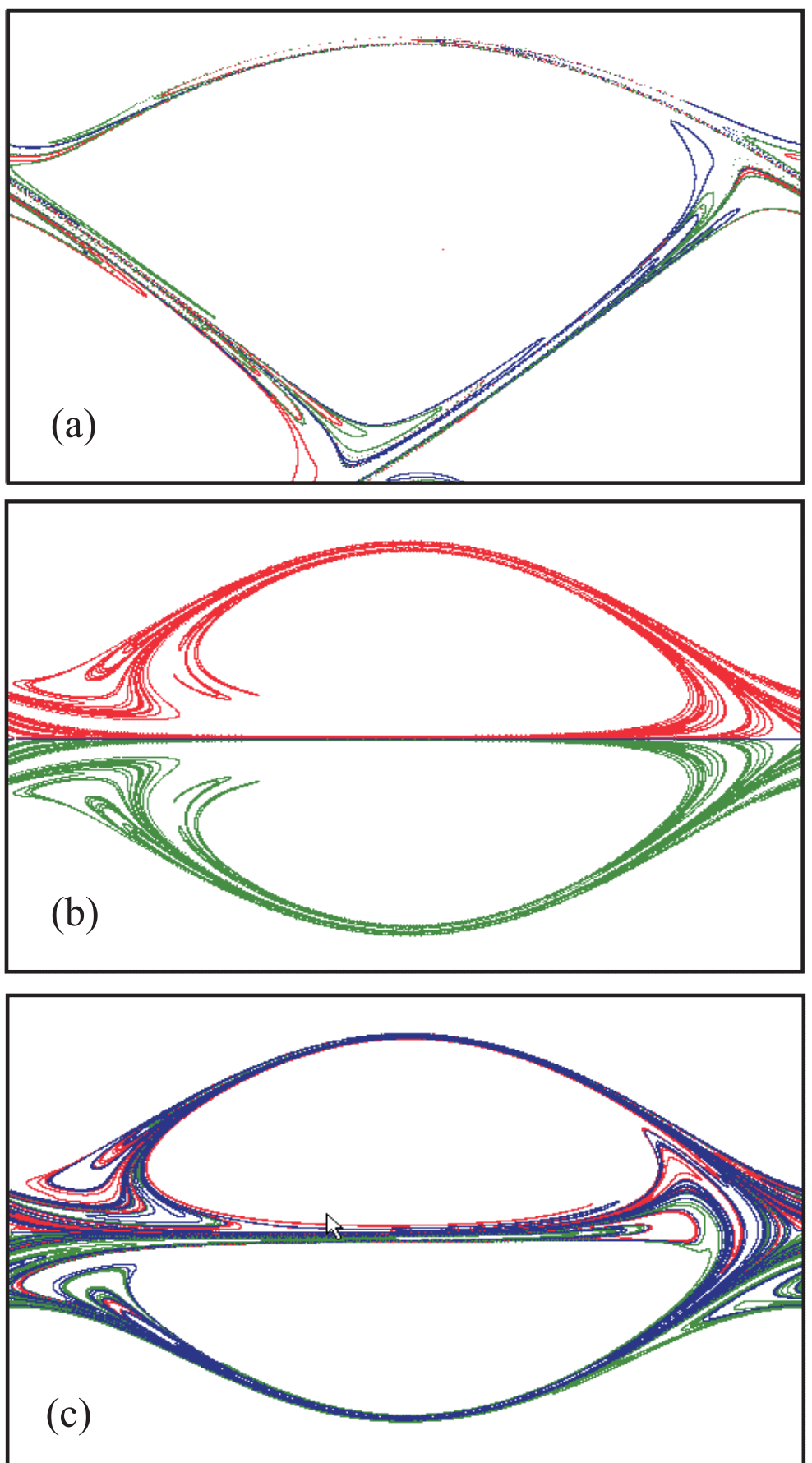

Figure 7: The evolution of the initial homoclinic and heteroclinic orbits after 3 intersections with the Poincaré section. Blue, red and green colour presents orbits (A),(B) and (C) respectively for the cases (a) Type I, (b) Type IIa and (c) Type IIb. 

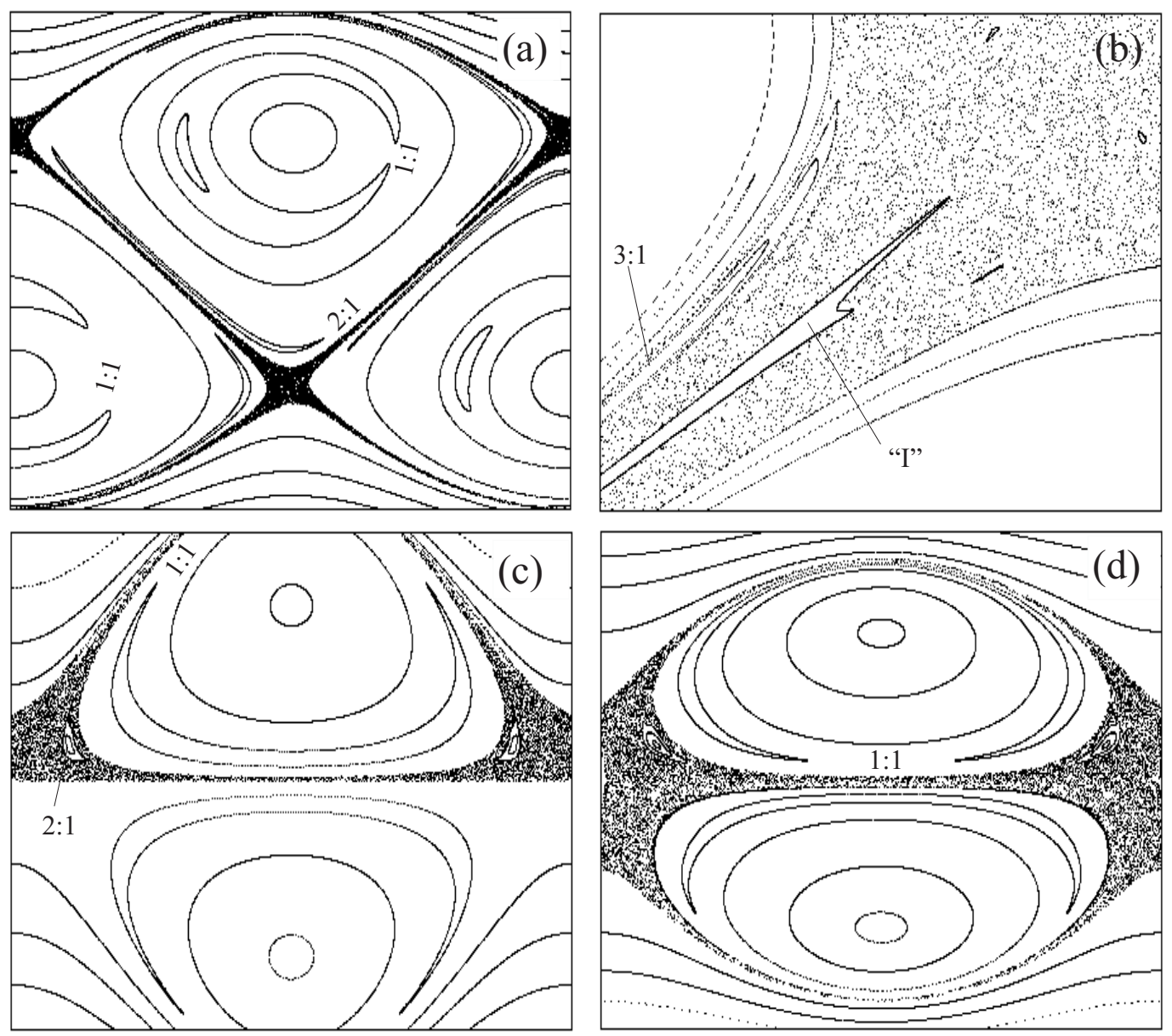

Figure 8: The Poincaré section for the examined systems and for $a=1$, $k=k_{\text {rec }}, \omega=1$ and $\varepsilon=0.01$. a) Type I, b) magnification of the area around the unstable fixed point at the center of (a), c) Type IIa, where only the upper chaotic region is shown, d) Type IIb. 